\title{
Japan's ERATO programme found to be working well
}

\section{Tokyo}

JAPAN's Exploratory Research for Advanced Technology (ERATO) programme was set up in 1981 as an experiment to try to break away from the rigid hierarchy of universities and stimulate the kind of creativity that many Japanese researchers think flourishes in the West. Last month, in its first-ever evaluation by a group of foreign researchers, many parts of ERATO pass with flying colours.

The greatest praise was reserved for the Quantum Magneto-Flux Logic Project (1986-91) led by Eichi Goto of Tokyo University, which is exploring the possibility of making ultra-fast computers using magnetic quanta. John Rowell of Bell Communications Research, who assessed the project, said he was amazed that such a "sense of rebellion" could be fostered within the confines of Japan's existing industrial and university structure.

He believes the project has achieved success, as exemplified by the demonstration of a variety of logic functions using single flux elements composed of Josephson junctions. Such progress could not have been made in the United States, he says, where expertise in Josephson junction technology has been allowed to "evaporate".

ERATO, which is run by the Science and Technology Agency's Research and Development Corporation, has financed 18 projects, most of them high-risk research with some potential for application. Two or three new projects are begun each year and funded for five years at \$2-3 million per year. Except for the project head, no member of the 15-20 person project teams may be over 35 years old. Researchers are drawn from industry and universities and include several foreigners.

The new evaluation comes from the Japan Technology Evaluation Program, which is supported largely by the US National Science Foundation. The Perfect Crystal Project (1981-86) headed by Junichi Nishizawa, Tohoku University, which developed improved methods for growing perfect crystals of silicon and gallium arsenide, was another project judged "very successful". Edward Wolf, director of the National Nannofabrication Facility at Cornell University, said he was particularly impressed with the effective coupling of the project with industry.

Among the biological projects, the Bioinformation Transfer Project (1983-88) headed by Osamu Hayaishi, Osaka Bioscience Institute, which examined prostaglandins in the central nervous system and their role in biological information transfer, is given high marks by Dale Oxender, director for molecular genetics at the
University of Michigan. Seven patents have been filed and the results may have important applications in the pharmaceutical industry, Oxender says.

Seen as successful, but less imaginative, was the Superbugs Project led by Koki Horikoshi of RIKEN, which aims at finding commercial applications for microorganisms that can thrive in extremes of $\mathrm{pH}$, temperature or salinity. The Bioholonics Project (headed by Denichi Mizuno, Teikyo University) and the Molecular Dynamic Assembly Project (H. Hotani,
Kyoto University) were seen as "comparable to similar efforts in the United States".

Nearly all evaluators noted that ERATO gives project leaders considerable freedom to spend money as they wish, in contrast to the bureaucratic regulations surrounding financial support from the Ministry of Education, Science and Culture, But university researchers who accept ERATO funds are almost invariably "cutoff" from Ministry of Education funds unless they are "politically powerful". And Rita Colwell, director of Maryland Biotechnology Institute, drew attention to the total lack of female scientists in ERATO and the lack of leadership opportunities for female scientists in Japan.

David Swinbanks

\section{AAU issues guidelines on dealing with scientific fraud}

\section{Washington}

NEw guidelines intended to help universities to find ways to "distinguish fraud from the honest error and the ambiguities of interpretation that are inherent in the scientific process" were issued last week by the Association of American Universities (AAU), a body that represents most big US research universities.

The guidelines come as public concern over scientific misconduct is increasing, carrying with it the possibility that the US Congress will introduce strict new legislation to control research. A key part of the thinking behind the guidelines is that "institutions ought to be held responsible

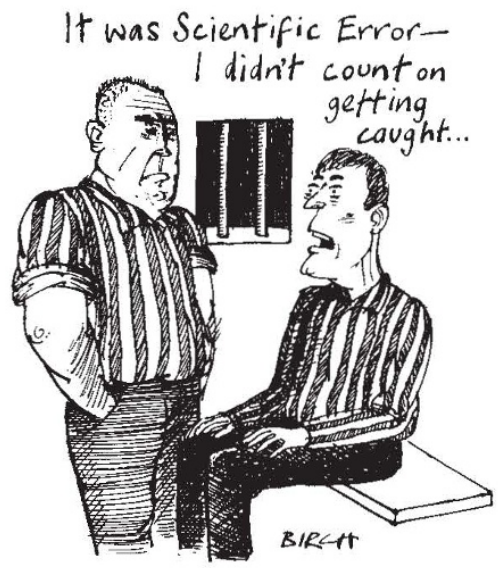

for the conduct of their faculty", according to Robert Rosenzweig, AAU president.

The new guidelines are simpler and stricter than draft guidelines issued by the Public Health Service (PHS) in 1986 and by the National Science Foundation in 1987. The guidelines do not accept that the resignation of a faculty member can close the books on a misconduct investigation, even though that action may provide an institution's simplest way to avoid public scandal. And once a conclusion has been reached, the institution should discharge its responsibilities to "the public, the sponsors of research, the scientific literature, and the scientific community".

The guidelines take up two of the commonest criticisms of misconduct investigations: that they take too long and that they can cause greater harm to the complainant than to the guilty.

A preliminary inquiry should be completed within 30 days and a full investigation, if warranted, within 120 days, although an institution may "acknowledge formally" that the nature of the case makes it impossible to meet the deadline. The guidelines acknowledge that "younger, less senior people are particularly vulnerable" when making accusations of misconduct, and urges that complainants be protected.

Little is made of another common criticism, that internal investigatory committees are inevitably under pressure to save face for the institution involved.

The new guidelines have been sent to all of AAU's 54 member institutions who will decide for themselves if they wish to use them as the basis for dealing with research fraud. AAU member institutions between them receive 60 per cent of all research grants given out by the National Institutes of Health (NIH), but the PHS, which is responsible for $\mathrm{NIH}$, has yet to complete its own guidelines. An official notice of "proposed rule making" was published four months ago, more than two years after initial guidelines were produced. The final regulations are unlikely to be ready before May.

Alun Anderson

\section{Erratum}

Contrary to a News item in Nature 337, 7; 1988 . it is Johann Deisenhofer who is now in Texas (at the Howard Hughes Institute, University of Texas Southwestern Medical Center, Dallas) and Hartmut Michel who has remained at a Max Planck Institute (in Frankfurt). 